\title{
Implantação da cantina escolar saudável em escolas do Distrito Federal, Brasil ${ }^{1}$
}

\author{
Implementation of healthy school canteens in \\ schools of the Federal District, Brazil
}

Nina Flávia de Almeida AMORIM²

Bethsáida de Abreu Soares SCHMITZ2

Maria de Lourdes Carlos Ferreirinha RODRIGUES²

Elisabetta Gioconda Iole RECINE²

Cristine Garcia GABRIEL ${ }^{3}$

\section{RE S U M O}

\section{Objetivo}

Desenvolver e aplicar uma metodologia para avaliar a implantação da cantina escolar saudável em escolas do Distrito Federal.

\section{Métodos}

Um estudo descritivo e avaliativo sobre uma intervenção educativa realizado em 2006, por meio de um curso de capacitação para proprietários de cantinas, em que foram avaliados a implantação dos Dez Passos da Cantina Escolar Saudável e o perfil de funcionamento da cantina em três momentos (antes do curso, seis meses e dois anos depois). Para avaliação das preparações, criou-se um sistema de classificação em mais ou menos saudáveis.

\section{Resultados}

Foram capacitados 35 proprietários de cantina; dentre eles, nove participaram dos três momentos de avaliação. Houve uma maior aplicação dos Dez Passos da Cantina Escolar Saudável e um maior oferecimento de lanches mais saudáveis do $1^{\circ}$ para o $2^{\circ}$ momento, entretanto o $3^{\circ}$ momento de avaliação não manteve o mesmo comportamento. Apesar da descontinuidade observada no processo de acompanhamento da intervenção, os participantes buscaram um maior contato com outras cantinas interessadas no desenvolvimento de uma cantina saudável $(p=0,002)$.

\footnotetext{
1 Artigo elaborado a partir da dissertação de NFA AMORIM, intitulada "Desenvolvimento e avaliação de uma proposta metodológica de implantação da lanchonete escolar saudável". Universidade de Brasília; 2010. Apoio: Ministério da Saúde (Convênio n 4626/2006)

2 Universidade de Brasília, Faculdade de Ciências da Saúde, Programa de Pós-Graduação em Nutrição Humana. Brasília, DF, Brasil.

${ }^{3}$ Universidade Federal de Santa Catarina, Centro de Ciências da Saúde, Departamento de Saúde Pública, Programa de Pós-Graduação em Saúde Coletiva. Campus Universitário, Trindade, 88040-970, Florianópolis, SC, Brasil. Correspondência para/Correspondence to: CG GABRIEL.E-mails: <cris_ntr@hotmail.com>; <criggabriel@yahoo.com.br>.
} 
204 | NFA AMORIM et al.

\section{Conclusão}

O curso de capacitação apresentou resultados positivos, principalmente a curto prazo, entretanto o processo de acompanhamento durante um ano para esse público não foi o suficiente para garantir a sustentabilidade das ações. Para a efetiva implantação e manutenção da cantina saudável é fundamental o acompanhamento sistemático desse processo, apoiado no envolvimento da comunidade escolar e em legislação específica.

Termos de indexação: Alimentação escolar. Capacitação. Educação alimentar e nutricional.

\section{A B S T R A C T}

\section{Objective}

A methodology for assessing the implementation of healthy school canteens in schools of the Federal District was developed and administered.

\section{Methods}

A descriptive study was done in 2006 to assess the following: a) an educational intervention that trained canteen owners and implemented ten steps to a healthy school canteen; and b) canteen profiles on three different occasions: before the training course and six and twenty-four months after. A system was then developed for classifying the healthiness of the preparations.

\section{Results}

Thirty-five canteen owners were trained and nine participated in all three canteen assessments. Compliance with the ten steps to a healthy school canteen and availability of healthier snacks increased from the first to the second assessment, but not to the third. Although canteen owners were no longer compliant with the intervention, they tried to stay more in touch with other canteens interested in offering healthy snacks $(p=0.002)$.

\section{Conclusion}

The training course had positive results, especially in the short run. However, the one-year follow-up for this group was not enough to ensure sustainability of the actions. Effective implementation and maintenance of healthy canteens require systematic monitoring and legal and scholastic support.

Indexing terms: School feeding. Training. Food and nutrition education.

\section{N T R O D U Ç Ã O}

Independentemente do ambiente em que está contextualizada, a alimentação envolve muitas dimensões intrínsecas ao ser humano, não sendo diferente dentro do espaço escolar. A escola deve permitir uma relação saudável com o alimento, respeitando e promovendo a cultura e as tradições alimentares locais, conduzindo à realização do direito humano à alimentação adequada e à segurança alimentar e nutricional. Destaca-se que a promoção da alimentação saudável dentro do ambiente escolar contribui também para o controle e a diminuição da alta prevalência de obesidade e sobrepeso, atualmente encontrada na população escolar ${ }^{1-3}$.

No ambiente escolar, o acesso aos alimentos pode ocorrer por meio de fontes diversas, sendo uma delas as cantinas escolares, presentes na maioria das escolas da América Latina ${ }^{4,5}$. Nesse sentido, a cantina comercial escolar deve ser considerada no planejamento de ações para a promoção da alimentação saudável no ambiente escolar, por ser um dos locais onde a prática alimentar se efetua, com oferta diária de alimentos ${ }^{5,6}$.

Estudos mostram que a maioria dos alimentos vendidos nas cantinas escolares apresenta excesso de açúcares, gorduras e sal7,8, mesmo quando da existência de instrumentos normativos que regulamentem os alimentos a serem vendidos nesses estabelecimentos ${ }^{5,9}$.

É necessário o desenvolvimento de uma cantina escolar saudável: ela deve ser um produtor de refeições e lanches de qualidade, que englobem aspectos nutricionais e higiênicos, que visem 
à segurança alimentar e nutricional do aluno e da comunidade escolar, e que respeitem o prazer e o hábito cultural ${ }^{10}$.

Para a realização do direito humano à alimentação adequada e da segurança alimentar e nutricional, é necessário um processo de informação e formação da comunidade escolar dentro de uma perspectiva de conquista da cidadania, incluindo os proprietários de cantinas escolares.

Entretanto, apesar da importância do tema e do estímulo contido em algumas diretrizes e políticas atuais brasileiras para a implantação de uma cantina escolar saudável ${ }^{10,11}$, poucas são as experiências desenvolvidas até o momento no País, o que dificulta um adequado delineamento de políticas públicas com as cantinas comerciais escolares $^{5,9}$.

No Distrito Federal, o Projeto A Escola Promovendo Hábitos Alimentares Saudáveis desenvolve, desde 2003, ações educativas com proprietários de cantinas escolares para a implantação da Cantina Escolar Saudável ${ }^{12}$. Em 2006, foi realizado um curso de capacitação e apoio técnico aos proprietários interessados ${ }^{13}$. Desse modo, este artigo tem por objetivo apresentar o desenvolvimento e a aplicação de uma metodologia para avaliar a implantação da Cantina Escolar Saudável entre os participantes desse curso.

\section{MÉ T O D O S}

Estudo descritivo e avaliativo sobre uma intervenção educativa, do tipo antes e depois, com cantinas comerciais escolares. A intervenção refere-se a um curso de capacitação específico para proprietários de cantinas escolares, que foi realizado no ano de 2006, com o objetivo de apoiar os proprietários na implantação da Cantina Escolar Saudável. Para acompanhamento da evolução de tais cantinas, foram desenvolvidos e aplicados dois instrumentos de avaliação dos estabelecimentos. Tal avaliação ocorreu uma vez antes e duas vezes após o curso de capacitação: seis meses e dois anos depois, sendo esta última avaliação realizada em 2008. Os resultados referentes à execução e à avaliação do curso de capacitação já se encontram publicados ${ }^{13}$, e os dados de avaliação e de acompanhamento da Cantina Escolar Saudável estão apresentados neste artigo.

A amostra de proprietários de cantinas escolares caracterizou-se por ser não probabilística, tendo em vista que o curso de capacitação foi aberto ao público interessado, e que não se fez uso de formas aleatórias de seleção. Todos os indivíduos interessados em participar foram inseridos no grupo de capacitação e, posteriormente, no de acompanhamento. Ao total, inscreveram-se no curso 41 participantes, representando 35 estabelecimentos comerciais, dentre os quais cinco eram cantinas de escolas públicas e 30 de escolas particulares. Apenas dois proprietários não concluíram o curso; durante os encontros de acompanhamento, participaram em média oito proprietários.

Para avaliar a implantação da Cantina Escolar Saudável foram desenvolvidos e aplicados dois instrumentos: A) Instrumento de avaliação dos Dez Passos da Cantina Escolar Saudável; B) Instrumento de avaliação do Perfil da Cantina Escolar Saudável.

O instrumento A coletou informações sobre a existência de cursos prévios de capacitação na área de alimentação e nutrição; sobre ações para a integração com a comunidade escolar e com outras cantinas; sobre ações de educação nutricional realizadas no estabelecimento; sobre desenvolvimento de estratégias para a promoção dos lanches saudáveis e sobre o aperfeiçoamento e o monitoramento da cantina escolar.

O instrumento B continha informações sobre os produtos comercializados, a produção e a qualidade dos alimentos utilizados, os conhecimentos do proprietário em relação a alguns distúrbios nutricionais dos alunos, a interferência da comunidade escolar na comercialização de alimentos e a presença de venda de alimentos ao redor da escola.

Conforme relatado, esses instrumentos foram aplicados com os proprietários das cantinas 
escolares em três momentos: antes do curso de capacitação (2006), entre o sexto e o oitavo mês de realização do curso (2006) e após dois anos do término do curso (2008).

Para análise e avaliação dos produtos comercializados nas cantinas escolares, foi elaborado um sistema de pontuação para a classificação desses alimentos em mais ou menos saudáveis. Para isso, foram realizadas as seguintes etapas:

1) Definição dos parâmetros técnicos para a classificação dos alimentos: Diante da literatura investigada e dos vários sistemas de classificação encontrados, escolheu-se o parâmetro técnico mais adequado ao contexto. Para tanto, recorreu-se à definição de alimento saudável do Departamento Americano Food and Drug Administration (FDA) e confrontou-se com a realidade observada. A FDA estabelece que o alimento saudável deve ter menos que $13 \mathrm{~g}$ de gordura total, menos que $4 \mathrm{~g}$ de gordura saturada, menos que $60 \mathrm{mg}$ de colesterol e menos que $360 \mathrm{mg}$ de sódio por quantidade de alimento normalmente consumida. Essa definição pode ser aplicada pelo tamanho da porção do produto ou por $50 \mathrm{~g}$ no caso de alimentos com quantidade de referência normalmente consumida de $30 \mathrm{~g}$ ou duas colheres de sopa (ou menos) $)^{14-16}$.

Além disso, o FDA estabelece que, para atender a definição de um alimento saudável, o alimento precisa conter um ou mais dos seguintes nutrientes: vitamina A, vitamina C, ferro, cálcio, proteína e fibra, em um valor igual ou maior que $10 \%$ da Dietary Reference Intake (DRI) para aquele nutriente, por porção normalmente consumida daquele alimento ${ }^{14-16}$. Para se concluir o processo de análise de $10 \%$ de recomendação de consumo diário desses nutrientes, definiram-se também os valores máximos (Tolerable Upper Intake Level - UL) para 10\% da UL para as vitaminas e minerais preconizados e $10 \%$ da faixa de distribuição aceitável (Acceptable Macronutrient Distribution Range - AMDR) para fibra e proteína.

Foram utilizadas, neste estudo, as faixas etárias de 4 a 8 anos e de 9 a 13 anos: faixas em que se encontram os alunos das escolas pesquisadas. Vale observar que a gordura total, a gordura saturada, o colesterol e o sódio não variaram de acordo com as faixas etárias.

2) Análise da composição nutricional dos alimentos: Inicialmente, para a avaliação da composição nutricional de todos os alimentos, utilizaram-se tabelas de composição de alimentos de uso comum nas pesquisas brasileiras, e quando o produto não estava nelas incluído, buscaram-se dados do seu rótulo.

3) Desenvolvimento de fichas técnicas de preparação dos alimentos elaborados no próprio estabelecimento, isto é, não industrializados: A fim de verificar o tamanho da porção ofertada e avaliar a composição nutricional desses alimentos/preparações de acordo com a realidade local, foram desenvolvidas fichas técnicas de preparação para alimentos/preparações que pudessem ser produzidos nas cantinas escolares. Para elaboração das referidas fichas, seguiram-se procedimentos de pesagem dos ingredientes/alimentos em uma balança da marca Scale ${ }^{\circledast}$ com capacidade de $3 \mathrm{~kg}$ e com precisão de $1 \mathrm{~g}$. A elaboração das referidas fichas foi realizada de acordo com a metodologia de Akutsu et al. ${ }^{17}$.

4) Elaboração do sistema de pontuação para a classificação dos alimentos em mais e menos saudáveis: Depois de estruturada a avaliação da composição nutricional dos alimentos, foi desenvolvida uma análise estatística com o intuito de criar um sistema de classificação por pontuação entre os alimentos ofertados nas cantinas, de forma a identificar cada um como mais ou menos saudável. Foram observadas em cada alimento as quantidades dos 10 nutrientes já descritos, dividindo-os em: bloco 1, composto por gordura total, gordura saturada, colesterol e sódio; e bloco 2, vitamina A, vitamina C, fibra, proteína, cálcio e ferro.

Posteriormente, foi verificado se os nutrientes do bloco 1 estavam todos com a quantidade abaixo do limite máximo da Recomendação 
Diária (RDA). Caso algum estivesse acima, o alimento seria classificado como "não adequado". Após o atendimento desses requisitos, o alimento passava para a análise do bloco 2. Nesse bloco, para que houvesse adequação com o recomendado pelo FDA, pelo menos um dos nutrientes deveria estar dentro do limite adequado, limitado inferiormente pela RDA e, superiormente, pela UL ou pelo limite superior da AMDR, dependendo do nutriente. Caso nenhum nutriente estivesse dentro dos limites estabelecidos, o alimento seria classificado como "não adequado".

No caso em que o alimento no bloco 1 estivesse com os valores de gordura total, gordura saturada, colesterol e sódio abaixo do limite máximo da RDA e estivesse no bloco 2 com algum dos nutrientes dentro do limite adequado, limitado inferiormente pela RDA e, superiormente, pela UL (nos casos da vitamina A, C, do cálcio e do ferro) e pelo limite superior da AMDR (no caso da proteína), ele seria classificado como "adequado".

Conforme dito anteriormente, para a montagem das pontuações foram consideradas as premissas do FDA para a adequação do alimento: se um alimento não atendesse os pré-requisitos para ser classificado, também não seria construída uma pontuação para ele.

Foram considerados dois valores para essa construção, sendo um para cada bloco. No bloco 1, foi atribuído o valor 1 positivo (+1) para os nutrientes que estavam com o valor abaixo do limite máximo da recomendação diária, e o valor 1 negativo (-1) para os nutrientes que estavam com quantidades acima da recomendação diária. Após essa valoração dos itens por bloco, calculou-se sua soma e dividiu-se pelo número de informações que se tinha no bloco 1. Dessa forma, a pontuação do bloco 1 tem um valor mínimo de -1 e um valor máximo de +1 .

Para o bloco 2, foi atribuído o valor 1 positivo (+1) para os nutrientes que estivessem de acordo com os requisitos desse bloco. Para os nutrientes que não cumprissem os requisitos esta- belecidos, foi dado um valor 0 . Diferentemente do que foi feito com relação aos requisitos do bloco 1, em que foram atribuídos valores negativos, isso não foi feito no bloco 2, pois, de acordo com a classificação do FDA, um só item do bloco 2 que não esteja adequado ao estabelecido não implica uma desclassificação total do alimento como "adequado", uma vez que apenas um dos seis nutrientes do bloco 2 necessita estar dentro dos requisitos para validar o bloco 2 .

Dessa forma, como no bloco 1, fez-se a soma dos pontos atribuídos e se dividiu pelo número de informações constantes no bloco 2 . Assim, levando em conta os valores atribuídos aos nutrientes do bloco 2, o valor mínimo da pontuação é de 0 (quando nenhum nutriente está dentro do intervalo determinado) e o valor máximo é de 1 (quando todos estão).

Após a criação do sistema de pontos, os alimentos foram classificados em mais ou menos saudáveis e foi realizada a análise em relação aos três momentos de avaliação, e em relação ao cumprimento ou não dos Dez Passos da Cantina Escolar Saudável. Optou-se por analisar os cinco alimentos/preparações com maiores e menores pontuações. No entanto, observou-se a existência de empate na pontuação e isso acarretou mais de cinco alimentos/preparações analisados em alguns casos.

Foram utilizados os seguintes testes não paramétricos com o intuito de demonstrar se houve diferença entre o desempenho das cantinas nos diferentes momentos da pesquisa: teste $\mathrm{Q}$ de Cochran, utilizado para verificar se três ou mais amostras diferiam significativamente entre si, e teste de mudança de McNemar, para verificar se duas amostras relacionadas eram diferentes. Em função da redução da amostra no terceiro momento de avaliação, foi necessário considerar um maior rigor estatístico para possibilitar a expansão da amostra para a população-alvo. Sendo assim, apesar de vários resultados terem obtido valores $p<0,05$, valor esse tradicionalmente aceito como relevância estatística, assumiu-se que houve dife- 
rença estatística apenas nos casos cujo erro do teste foi inferior a $1 \%(p<0,01)$.

A presente pesquisa foi aprovada pelo Comitê de Ética em Pesquisa com Seres Humanos da Faculdade de Saúde - Universidade de Brasília (processo $\mathrm{n}^{\circ}$ 006/2005), tendo todos os participantes assinado um termo de consentimento livre e esclarecido, de acordo com as normas estabelecidas pela Resolução 196/96 do Conselho Nacional de Saúde.

\section{RES U LTA D O S}

Dentre as 35 cantinas escolares integrantes do curso de capacitação, 24 participaram da $1^{\text {a }}$ avaliação (antes do curso de capacitação), sendo três $(12,5 \%)$ cantinas de escolas públicas e 21 $(87,5 \%)$ de escolas particulares; na $2^{a}$ avaliação (seis meses após o curso), participaram 18 cantinas, sendo duas de escolas públicas $(11,0 \%)$ e $16(89,0 \%)$ de escolas particulares; e na $3^{\mathrm{a}}$ avaliação (dois anos após o curso), houve participação de representantes de nove cantinas, todas de escolas particulares. Os motivos para a recusa foram a impossibilidade de contato, a mudança de proprietário e a falta de interesse na participação. Os dados apresentados neste artigo relativos à comparação nos três momentos de avaliação dizem respeito às últimas nove cantinas de escolas particulares citadas.

A análise do perfil dos proprietários das cantinas participantes identificou que $100,0 \%$ era do sexo feminino, predominantemente com idade acima de 35 anos (67,0\%), tendo a maioria o ensino fundamental completo $(56,0 \%)$ como escolaridade (dados não apresentados em Tabelas).

Verificou-se um aumento percentual na presença de bar, padaria e ambulantes ao redor da escola do $1^{\circ}$ para o $3^{\circ}$ momento de avaliação, variando neste último de 33,0\% a 44,0\%. Na $3^{\text {a }}$ avaliação, 100,0\% dos entrevistados referiram que as escolas não permitiam a entrada de alimentos desses estabelecimentos no espaço escolar. Além disso, observou-se nos três momentos interferência da direção da escola quanto à suges- tão ou restrição de algum tipo de lanche ou produto alimentício na cantina escolar (dados não apresentados em Tabelas).

Houve um aumento percentual de cantinas que não vendiam alimentos industrializados com teor de gordura saturada superior a 10\% do valor energético total (percentuais na $1^{\mathrm{a}}, 2^{\mathrm{a}}$ e $3^{\mathrm{a}}$ avaliação foram $11 \%$, 22\% e $56 \%$ respectivamente), bem como de cantinas que não comercializavam alimentos com gordura vegetal hidrogenada (percentuais na 1 $1^{\mathrm{a}} 2^{\mathrm{a}}$, e $3^{\mathrm{a}}$ avaliação foram $11 \%, 11 \%$ e $67 \%$, respectivamente). Por outro lado, houve uma diminuição do percentual de cantinas que ofertavam porções diferenciadas de salgados segundo a faixa etária (percentuais na $1^{a}, 2^{a}$, e $3^{a}$ avaliação foram $22 \%, 33 \%$ e $11 \%$, respectivamente) (dados não apresentados em Tabelas).

Observou-se um aumento percentual de dez dentre os treze itens relativos ao cumprimento dos passos para a implantação da Cantina Escolar Saudável, do $1^{\circ}$ para o $2^{\circ}$ momento de avaliação. Por outro lado, do $2^{\circ}$ para o $3^{\circ}$ momento, houve redução do percentual de cumprimento de itens referentes aos passos 2, 3, 5, 6, 7, 8 e 10. Dentre esses sete passos, dois itens, que integravam o passo 5 - exposição de lanches mais saudáveis $(p=0,002)$ e redução ou retirada de alimentos ricos em açúcares e gorduras $(p=0,006)$ - mostraram um menor cumprimento na aplicação desses passos no $3^{\circ}$ momento da avaliação (Tabela 1).

O percentual das cantinas que participaram de outros cursos sobre alimentação e nutrição (passo 1) aumentou ao longo do processo de avaliação $(p=0,05)$. Esse mesmo comportamento foi observado para o passo 9: aumentou o contato com outras cantinas interessadas no desenvolvimento de uma cantina escolar mais saudável $(p=0,002)$.

Como outra forma de avaliação da implantação da Cantina Escolar Saudável, os alimentos comercializados foram então classificados em mais ou menos saudáveis. A Tabela 2 apresenta a classificação dos alimentos e seu percentual de oferta nas cantinas durante os três momentos de 
Tabela 1. Acompanhamento da realização da implantação dos Dez Passos da Cantina Escolar Saudável. Brasília (DF), $2006-2008$.

\begin{tabular}{|c|c|c|c|c|c|c|c|}
\hline \multirow{2}{*}{ Passos para a implantação da cantina escolar saudável } & \multicolumn{2}{|c|}{$1^{\mathrm{a}}$ aplicação } & \multicolumn{2}{|c|}{$2^{a}$ aplicação } & \multicolumn{2}{|c|}{$3^{a}$ aplicação } & \multirow{2}{*}{$p$-valor } \\
\hline & $\mathrm{n}$ & $\%$ & $\mathrm{n}$ & $\%$ & $\mathrm{n}$ & $\%$ & \\
\hline \multicolumn{8}{|l|}{ Passo 1} \\
\hline Participação em outros cursos sobre alimentação e nutrição & 2 & 22,2 & 3 & 33,3 & 7 & 77,8 & 0,050 \\
\hline \multicolumn{8}{|l|}{ Passos 2 e 10} \\
\hline Pedido de apoio à escola para implantação da cantina saudável & 4 & 44,4 & 8 & 88,9 & 1 & 11,1 & 0,016 \\
\hline $\begin{array}{l}\text { Pedido de apoio aos educadores para implantação da cantina saudá- } \\
\text { vel }\end{array}$ & 7 & 77,8 & 8 & 88,9 & 2 & 22,2 & 0,021 \\
\hline \multicolumn{8}{|l|}{ Passo 3} \\
\hline Contato com pais sobre a implantação da cantina saudável & 7 & 77,8 & 7 & 77,8 & 3 & 33,3 & 0,135 \\
\hline \multicolumn{8}{|l|}{ Passo 5} \\
\hline Realização de promoções/barateamento de lanches saudáveis & 2 & 22,2 & 6 & 66,7 & 6 & 66,7 & 0,069 \\
\hline Exposição de lanches mais saudáveis & 8 & 88,9 & 9 & 100,0 & 1 & 11,1 & 0,002 \\
\hline Redução ou retirada de alimentos ricos em açúcares e gorduras & 8 & 100,0 & 7 & 77,8 & 2 & 22,2 & 0,006 \\
\hline \multicolumn{8}{|l|}{ Passos 6 e 7} \\
\hline $\begin{array}{l}\text { Realização de pesquisa com os alunos sobre o que gostariam de co- } \\
\text { mer na cantina }\end{array}$ & 5 & 55,6 & 7 & 77,8 & 4 & 44,4 & 0,311 \\
\hline $\begin{array}{l}\text { Realização de pesquisa sobre a opinião dos alunos em relação à can- } \\
\text { tina }\end{array}$ & 6 & 66,7 & 7 & 77,8 & 3 & 33,3 & 0,156 \\
\hline \multicolumn{7}{|l|}{ Passo 8} & 0,882 \\
\hline Realização de atividades para promoção de um lanche mais saudável & 3 & 33,3 & 7 & 77,8 & 6 & 66,7 & 0,156 \\
\hline $\begin{array}{l}\text { Uso do espaço da cantina escolar com materiais educativos sobre } \\
\text { alimentação e nutrição }\end{array}$ & 3 & 33,3 & 7 & 77,8 & 3 & 33,3 & 0,135 \\
\hline \multicolumn{8}{|l|}{ Passo 9} \\
\hline $\begin{array}{l}\text { Contato com outras cantinas que tenham o objetivo de uma cantina } \\
\text { mais saudável }\end{array}$ & 1 & 11,1 & 2 & 22,2 & 8 & 88,9 & 0,002 \\
\hline
\end{tabular}

Nota: o passo 4, referente a "promover cursos para manutenção das boas práticas de manipulação de alimentos", foi analisado por meio da aplicação da lista de verificação das boas práticas de manipulação e fabricação de alimentos, de acordo com a RDC $n^{\circ} 216$. Agência Nacional de Vigilância Sanitária (15/set/2004).

avaliação. Na faixa etária de quatro a oito anos, cinco alimentos foram classificados como mais saudáveis (alimentos com as maiores pontuações), e seis como menos saudáveis (menores pontuações). Na faixa etária de nove a treze anos, foram sete alimentos mais saudáveis e seis menos saudáveis. Não se observaram diferenças significantes nos percentuais de oferecimento dos alimentos nos três momentos de avaliação.

Dentre os mais saudáveis para ambas as faixas etárias, a salada de fruta, o salgado assado, o bolo e o iogurte tiveram os maiores percentuais de oferecimento nos três momentos de avaliação. Na faixa etária de quatro a oito anos, os alimentos mais saudáveis e os menos saudáveis foram, res- pectivamente, a salada de fruta e o salgado assado e a pipoca de micro-ondas e o salgado folhado. Na faixa etária de nove a treze anos, destacaram-se como mais saudáveis o hambúrguer elaborado, a salada de fruta, o salgado assado e a vitamina, sendo também considerado como menos saudável o salgado folhado. Apesar de ter sido considerado um alimento mais saudável nas duas faixas etárias, o hambúrguer elaborado esteve presente em poucas cantinas escolares nas três aplicações (11\%).

Dentre os alimentos classificados como menos saudáveis, destaca-se nas duas faixas etárias o biscoito recheado, salgado frito, sanduíche natural, pipoca de micro-ondas e salgado folhado. 
210 | NFA AMORIM et al.

Tabela 2. Classificação dos alimentos mais e menos saudáveis ofertados pelas cantinas escolares em três momentos de acompanhamento, segundo faixa etária. Brasília (DF), 2006-2008.

\begin{tabular}{|c|c|c|c|c|c|}
\hline \multicolumn{6}{|c|}{ Faixa etária - 4 a 8 anos } \\
\hline & Pontuação & $1^{\text {a }}$ Aplicação (\%) & 2a Aplicação (\%) & 3a Aplicação (\%) & $p$-valor \\
\hline \multicolumn{6}{|l|}{ Alimentos com maiores pontuações } \\
\hline Salada de fruta & 0,90 & 88,9 & 88,9 & 77,8 & 0,779 \\
\hline Salgado assado & 0,90 & 88,9 & 100,0 & 100,0 & 0,368 \\
\hline Chá & 0,86 & 33,3 & 33,3 & 44,4 & 0,717 \\
\hline $\begin{array}{l}\text { Hambúrguer elaborado (pão, hambúrguer, queijo, } \\
\text { tomate e alface) }\end{array}$ & 0,86 & 11,1 & 11,1 & 11,1 & 1,000 \\
\hline Vitamina & 0,86 & 11,1 & 44,4 & 33,3 & 0,097 \\
\hline \multicolumn{6}{|l|}{ Alimentos com menores pontuações } \\
\hline Biscoito recheado & 0,38 & 11,1 & 0,0 & 0,0 & 0,368 \\
\hline Salgadinho em pacote (tipo Elma chips) & 0,38 & 22,2 & 22,2 & 55,6 & 0,050 \\
\hline Salgado frito & 0,38 & 11,1 & 33,3 & 55,6 & 0,135 \\
\hline Sanduíche natural & 0,38 & 55,6 & 77,8 & 77,8 & 0,368 \\
\hline Pipoca de micro-ondas & 0,29 & 22,2 & 11,1 & 11,1 & 0,368 \\
\hline Salgado folhado & 0,29 & 0,0 & 0,0 & 11,1 & 0,368 \\
\hline \multicolumn{6}{|c|}{ Faixa etária - 9 a 13 anos } \\
\hline \multicolumn{6}{|l|}{ Alimentos com maiores pontuações } \\
\hline $\begin{array}{l}\text { Hambúrguer elaborado (pão, hambúrguer, queijo, } \\
\text { tomate e alface) }\end{array}$ & 0,86 & 11,1 & 11,1 & 11,1 & 1,000 \\
\hline Salada de fruta & 0,86 & 88,9 & 88,9 & 77,8 & 0,779 \\
\hline Salgado assado & 0,86 & 88,9 & 100,0 & 100,0 & 0,368 \\
\hline Vitamina & 0,86 & 11,1 & 44,4 & 33,3 & 0,097 \\
\hline Bolo & 0,81 & 44,4 & 55,6 & 66,7 & 0,223 \\
\hline Chá & 0,81 & 33,3 & 33,3 & 44,4 & 0,717 \\
\hline logurte & 0,81 & 44,4 & 55,6 & 44,4 & 0,607 \\
\hline \multicolumn{6}{|l|}{ Alimentos com menores pontuações } \\
\hline Chocolate/bombons & 0,38 & 66,7 & 66,7 & 77,8 & 0,717 \\
\hline Sanduíche natural & 0,38 & 55,6 & 77,8 & 77,8 & 0,368 \\
\hline Salgado frito & 0,33 & 11,1 & 33,3 & 55,6 & 0,135 \\
\hline Biscoito recheado & 0,33 & 11,1 & 0,0 & 0,0 & 0,368 \\
\hline Pipoca de micro-ondas & 0,29 & 22,2 & 11,1 & 11,1 & 0,368 \\
\hline Salgado folhado & 0,24 & 0,0 & 0,0 & 11,1 & 0,368 \\
\hline
\end{tabular}

Entretanto, dentre esses, observou-se baixo percentual de oferecimento de biscoito recheado, pipoca e salgado folhado nos três momentos de avaliação. Os alimentos menos saudáveis mais ofertados foram salgadinho em pacote, sanduíche natural, chocolates e bombons e salgados fritos, mostrando um aumento percentual entre os momentos de avaliação.

A Tabela 3 indica o percentual médio de alimentos mais e menos saudáveis, nos três momentos de avaliação, segundo o cumprimento dos passos de implantação da Cantina Escolar Saudável. Observou-se que em todos os três momentos de avaliação, as cantinas que aplicaram os Dez
Passos da Cantina Saudável tiveram um percentual médio de oferta de alimentos mais saudáveis superior ao de alimentos menos saudáveis, com exceção apenas do passo 9 na $1^{\text {a }}$ avaliação e do passo 8 na última avaliação. Essa diferença percentual favorável ao oferecimento de alimentos mais saudáveis foi significante em vários passos, principalmente no $2^{\circ}$ momento de avaliação. A observação do percentual médio de alimentos mais saudáveis nos três momentos indicou um aumento desse percentual do $1^{\circ}$ para o $2^{\circ}$ momento, em todos os passos avaliados. Do $2^{\circ}$ para o $3^{\circ}$ momento, esse aumento percentual se deu em alguns passos. 
Tabela 3. Percentual médio de alimentos mais saudáveis (\%MaS) e de alimentos menos saudável (\%MeS) de acordo com a implantação dos Dez Passos da Cantina Escolar Saudável. Brasília (DF), 2006-2008.

\begin{tabular}{|c|c|c|c|c|c|c|c|c|c|c|c|c|}
\hline \multirow{2}{*}{$\begin{array}{l}\text { Dez Passos da Cantina } \\
\text { Escolar Saudável }\end{array}$} & \multicolumn{4}{|c|}{ Primeira avaliação } & \multicolumn{4}{|c|}{ Segunda avaliação } & \multicolumn{4}{|c|}{ Terceira avaliação } \\
\hline & $n$ & $\% \mathrm{MaS}$ & $\% \mathrm{MeS}$ & $p$-valor & $\mathrm{n}$ & $\% \mathrm{MaS}$ & $\% \mathrm{MeS}$ & $p$-valor & $n$ & $\% \mathrm{MaS}$ & $\% \mathrm{MeS}$ & $p$-valor \\
\hline \multicolumn{13}{|l|}{ Passo 1} \\
\hline $\begin{array}{l}\text { Participação em outros cur- } \\
\text { sos sobre alimentação* }\end{array}$ & 2 & 42,9 & 14,3 & - & 3 & 57,1 & 23,8 & 0,073 & 7 & 57,1 & 46,9 & 0,334 \\
\hline \multicolumn{13}{|l|}{ Passo 2 e 10} \\
\hline $\begin{array}{l}\text { Pedido de apoio à escola } \\
\text { para implantação da can- } \\
\text { tina saudável }\end{array}$ & 4 & 46,4 & 25,0 & 0,182 & 8 & 55,4 & 32,1 & 0,006 & 1 & 57,1 & 42,9 & - \\
\hline $\begin{array}{l}\text { Pedido de apoio aos edu- } \\
\text { cadores para implantação } \\
\text { da cantina saudável }\end{array}$ & 7 & 49,0 & 28,6 & 0,047 & 8 & 53,6 & 30,4 & 0,006 & 2 & 42,9 & 35,7 & 0,500 \\
\hline \multicolumn{13}{|l|}{ Passo 3} \\
\hline $\begin{array}{l}\text { Contato com pais sobre a } \\
\text { implantação da cantina } \\
\text { saudável }\end{array}$ & 7 & 49,0 & 22,4 & 0,004 & 7 & 57,1 & 32,7 & 0,011 & 3 & 57,1 & 47,6 & 0,691 \\
\hline \multicolumn{13}{|l|}{ Passo 5} \\
\hline $\begin{array}{l}\text { Realização de promoções } \\
\text { de lanches saudáveis }\end{array}$ & 3 & 42,9 & 19,0 & 0,199 & 7 & 59,2 & 30,6 & 0,006 & 6 & 52,4 & 35,7 & 0,110 \\
\hline $\begin{array}{l}\text { Exposição de lanches mais } \\
\text { saudáveis }\end{array}$ & 8 & 44,6 & 28,6 & 0,051 & 9 & 55,6 & 30,2 & 0,002 & 1 & 71,4 & 28,6 & - \\
\hline $\begin{array}{l}\text { Redução ou retirada de ali- } \\
\text { mentos ricos em açúcares } \\
\text { e gorduras } \\
\text { Passo } 6 \text { e } 7\end{array}$ & 8 & 46,4 & 28,6 & 0,049 & 7 & 55,1 & 34,7 & 0,016 & 3 & 57,1 & 57,1 & 1,000 \\
\hline $\begin{array}{l}\text { Realização de pesquisa } \\
\text { com alunos sobre preferên- } \\
\text { cias na cantina }\end{array}$ & 5 & 51,4 & 34,3 & 0,208 & 7 & 53,1 & 32,7 & 0,016 & 4 & 57,1 & 46,4 & 0,547 \\
\hline $\begin{array}{l}\text { Pesquisa sobre a opinião } \\
\text { dos alunos em relação à } \\
\text { cantina }\end{array}$ & 6 & 47,6 & 31,0 & 0,110 & 7 & 57,1 & 32,7 & 0,011 & 3 & 52,4 & 42,9 & 0,529 \\
\hline $\begin{array}{l}\text { Pesquisa sobre a opinião } \\
\text { dos pais em relação à can- } \\
\text { tina }\end{array}$ & 5 & 42,9 & 25,7 & 0,178 & 4 & 53,6 & 25,0 & 0,041 & 4 & 57,1 & 53,6 & 0,789 \\
\hline Passo 8 & & & & & & & & & & & & \\
\hline $\begin{array}{l}\text { Realização de atividades } \\
\text { para promoção de um lan- } \\
\text { che saudável }\end{array}$ & 3 & 42,9 & 19,0 & 0,199 & 7 & 57,1 & 32,7 & 0,011 & 6 & 52,4 & 40,5 & 0,224 \\
\hline $\begin{array}{l}\text { Uso do espaço da cantina } \\
\text { com materiais educativos }\end{array}$ & 3 & 52,4 & 28,6 & 0,038 & 7 & 55,1 & 30,6 & 0,011 & 3 & 47,6 & 57,1 & 0,529 \\
\hline Passo 9 & & & & & & & & & & & & \\
\hline $\begin{array}{l}\text { Contato com outras canti- } \\
\text { nas que objetivem uma } \\
\text { cantina saudável }^{*}\end{array}$ & 1 & 42,9 & 57,1 & - & 2 & 57,1 & 50,0 & 0,500 & 8 & 53,6 & 42,9 & 0,285 \\
\hline
\end{tabular}

* Não foi possível calcular o teste $t$ por falta de observações. 


\section{DISCUSS Ã O}

A alimentação escolar, incluindo os alimentos ofertados pela cantina comercial escolar, tem um papel fundamental na segurança alimentar e nutricional e na realização do direito humano à alimentação adequada dos escolares ${ }^{6,9}$. Dessa forma, algumas diretrizes nacionais subsidiam o desenvolvimento de ações e estratégias para a promoção da alimentação saudável na escola, incluindo recomendações para o desenvolvimento de uma cantina escolar saudável2,10,18.

No presente estudo, observou-se que dentre os 35 participantes do curso de capacitação, 26 (74,3\%) não participaram integralmente do processo de acompanhamento da implantação da Cantina Escolar Saudável ao longo dos dois anos de estudo, sendo os principais motivos para isso o desinteresse, a perda de contato e a mudança de proprietário na cantina. A elevada perda dos participantes durante o acompanhamento pode ser considerada como uma limitação do estudo. Destacam-se como fatores prejudiciais à permanência no estudo as modificações ocorridas no Distrito Federal referentes à legislação da venda de alimentos nas cantinas escolares. A existência de uma exigência normativa sobre a comercialização de alimentos foi um fator de atração para a participação no curso. Posteriormente, o veto governamental a essa mesma normativa ${ }^{11}$ desmotivou a continuidade do processo de acompanhamento, o que indica um comportamento instável desses proprietários de cantina diante da presença ou ausência de legislação específica. Em decorrência disso, o pequeno número de cantinas participantes nos três momentos foi um fator limitante das análises estatísticas realizadas. Entretanto, em trabalhos de campo dessa natureza, deve-se estar preparado para esse tipo de perda, principalmente considerando ações com extenso período de acompanhamento.

As legislações relacionadas à regulamentação da venda de alimentos em cantinas escolares objetivam o fortalecimento das políticas públicas voltadas para a área da alimentação e nutrição e para a garantia da segurança alimentar e nutricional e do direito humano à alimentação adequada9-11. Atualmente, tais legislações abarcam três aspectos importantes: a proibição da venda de determinados alimentos, o desenvolvimento de ações educativas, e as ações de fiscalização e sanções, não sendo abordada a capacitação dos proprietários de cantinas comerciais escolares, salvo exceções ${ }^{11}$.

Considerando-se o grau de escolaridade encontrado neste estudo (56\% da amostra têm apenas o primeiro grau completo), verifica-se que o desenvolvimento do processo educativo tornase importante para o favorecimento do espaço da cantina escolar como um local de promoção da alimentação saudável. Publicação anterior com essa mesma clientela demonstrou que os participantes do curso tiveram um bom desempenho com relação ao teste realizado antes e após o curso de capacitação, revelando ampliação de seus conhecimentos, além de terem avaliado o curso de forma positiva ${ }^{13}$.

Outro item a destacar é a presença de alimentos normalmente prontos para o consumo nos arredores da escola, o que pode comprometer as mudanças desenvolvidas dentro do ambiente escolar para a promoção da alimentação saudável, pré-dispondo o consumo inadequado pelos escolares $^{9,19}$. Os resultados deste estudo mostraram que o percentual de presença do comércio de alimentos ao redor da escola foi alto. Quanto ao tipo de alimento vendido nesses estabelecimentos, eles normalmente são ricos em açúcares, gorduras e sal9 ${ }^{9}$. Além da presença desse comércio, sabe-se que em alguns casos há a permissão da escola para entrada dos alimentos vendidos fora, o que compromete a promoção da alimentação saudável na escola. Esses alimentos podem competir com os ofertados por programas de alimentação do governo ou pelas cantinas. No caso das cantinas, isso pode diminuir a lucratividade desse estabelecimento e gerar desmotivação para a continuação da implantação da Cantina Escolar Saudável.

O emprego da alimentação saudável no ambiente escolar tem sido estimulado de várias 
formas e é uma tendência mundia|20,21. Nesse sentido, a legislação existente anteriormente no Distrito Federal tinha como um dos focos principais a proibição da venda de alimentos industrializados com teor de gordura saturada superior a $10 \%$ do valor energético total do produto, e de alimentos com gordura vegetal hidrogenada na sua composição ${ }^{11}$. Com relação ao primeiro, verificou-se, no curso, dificuldade no entendimento de como calcular o percentual de gordura saturada, e, com relação ao segundo, a constatação pelos participantes da presença disseminada da gordura vegetal hidrogenada em alimentos industrializados e do entendimento com relação aos seus malefícios para a saúde.

Observa-se que as legislações sobre o assunto apresentam as informações técnicas em uma linguagem de difícil entendimento pelos proprietários das cantinas, reforçando a necessidade de um processo educativo que torne compreensíveis, na prática, os preceitos legais. Com o processo educativo desenvolvido neste estudo, observou-se uma tendência à diminuição da oferta de alimentos ricos em gorduras saturadas (77,8\% para $44,4 \%$ ) e em gordura vegetal hidrogenada $(66,7 \%$ para $33,3 \%)$ do $1^{\circ}$ para o $3^{\circ} \mathrm{mo-}$ mento de avaliação, apesar de não ter sido estatisticamente significativo.

Concomitantemente ao processo educativo, é importante o desenvolvimento de ações de avaliação que verifiquem as dificuldades de implantação das diretrizes sobre alimentação e nutrição no ambiente escolar ${ }^{22}$. Dessa forma, a proposição dos Dez Passos da Cantina Escolar Saudável apresenta-se como uma sugestão de avaliação, juntamente com o instrumento de avaliação do Perfil de Funcionamento das cantinas escolares, podendo contribuir para o processo de implantação da Cantina Escolar Saudável, destacando-se a possibilidade de adaptação a distintas realidades. Gabriel et al. ${ }^{9}$ utilizaram esses mesmos dois instrumentos para a construção dos questionários aplicados na pesquisa realizada na região Sul do Brasil, referente à primeira avaliação da legislação sobre a venda de alimentos em cantinas comerciais escolares do estado de Santa Catarina.

De acordo com os resultados apresentados neste estudo, verificaram-se uma tendência de cumprimento dos Dez Passos da Cantina Escola Saudável bem como um oferecimento maior de alimentos considerados mais saudáveis com relação a alimentos menos saudáveis nas cantinas que aplicaram os Dez Passos da Cantina Escolar Saudável. Isso ocorreu após a realização do processo de acompanhamento com as cantinas, deflagrando, portanto, o início de um processo de formação crítica e de maior atenção para a necessidade de articulação e debate com os proprietários de cantina com relação à implantação de uma cantina escolar saudável. Segundo Sharman ${ }^{21}$, o desenvolvimento de um processo de capacitação e de acompanhamento do grupo de intervenção é importante para a sustentabilidade das ações.

Apesar disso, no $3^{\circ}$ momento de avaliação, verificou-se um enfraquecimento da tendência anteriormente destacada, o que evidencia que o grupo ainda necessitava da continuação do processo educativo iniciado, e que, provavelmente, as ações de acompanhamento deveriam ser executadas em um espaço de tempo menor do que o previsto nesta pesquisa. Há, ainda, a necessidade de desenvolvimento de ações de promoção da alimentação saudável com toda a escola, envolvendo a cantina no contexto maior do espaço escolar ${ }^{13}$.

Entretanto, observou-se que, apesar da descontinuidade observada no processo de acompanhamento da intervenção, os participantes buscaram um maior contato com outras cantinas com o objetivo de tentar consolidar o processo iniciado: resultado interessante no tocante à possibilidade da criação de uma rede de cantinas escolares saudáveis, devendo, portanto, ser estimulado com vistas ao fortalecimento de ações voltadas para criação e implantação de uma política pública que inclua a cantina escolar.

Com relação à classificação dos alimentos desenvolvida na pesquisa, ela mostrou-se impor- 
tante no sentido de discriminar, com base em critérios nutricionais, os alimentos usualmente ofertados em cantinas comerciais escolares, tentando identificar aqueles mais ou menos saudáveis. Destaca-se que, de forma geral, a classificação e os resultados obtidos em relação aos itens alimentares estão em acordo com as recomendações sobre a alimentação saudável no Brasil23. As exceções dizem respeito ao sanduíche natural, classificado como menos saudável, e ao hambúrguer elaborado, classificado como mais saudável. Estes resultados divergem das usuais recomendações técnicas na área de nutrição, confrontando principalmente com o conhecimento popular. No entanto, vale destacar que para o sanduíche natural não foi possível elaborar a FTP, uma vez que ele não era produzido nas cantinas avaliadas. Dessa forma, procedeu-se à verificação dos ingredientes nos rótulos dos sanduíches, observando-se que todos continham maionese: o excesso de gordura pode ter contribuído para o resultado encontrado. Quanto aos hambúrgueres, eles eram preparados nas cantinas e tiveram sua FTP calculada. Alguns pontos podem ter colaborado para a classificação desse alimento como mais saudável, a saber: não houve fritura com óleo em nenhum dos estabelecimentos, tendo sido utilizado chapa para cocção, sem adição de óleo. Além disso, todos os hambúrgueres continham salada de alface e tomate, o que favoreceu para a classificação desse alimento como mais saudável. Outro item que chamou a atenção foi a ausência do refrigerante dentre os principais alimentos menos saudáveis. Apesar disso, outros estudos indicam seu baixo valor nutricional, com elevados teores de açúcar que podem estar associados ao ganho de peso inadequado, principalmente na população infantil ${ }^{24,25}$.

Segundo Labouze ${ }^{26}$, a eficiência da classificação de alimentos entre mais e menos saudáveis é maior do que em "alimentos saudáveis" e "alimentos não saudáveis". De fato, considerando que atualmente existe uma grande opção de alimentos, tanto em termos quantitativos quanto qualitativos, bem como a importância de se garantir o respeito à cultura alimentar local e a segurança alimentar e nutricional, verifica-se que uma pontuação de alimentos entre mais e menos saudáveis poderá ser mais bem incorporada e aproveitada pelos proprietários de cantinas, contribuindo para a promoção da saúde e da alimentação saudável na escola.

Nesse sentido, vale lembrar as reflexões propostas por Vieira-da-Silva \& Almeida Filho ${ }^{27}$, que problematizam a construção social dos atuais conceitos de saúde e de promoção da saúde. Esses autores defendem que as políticas de saúde devem atender e priorizar as necessidades humanas, sendo essa uma estratégia essencial para formas equânimes e saudáveis de vida em sociedade. Os autores ainda reforçam que "priorizar necessidades não significa impor necessidades, porém definir o padrão tecnicamente aceitável, interagindo com as expectativas dos diversos modos de vida dos diferentes grupos sociais". Essas reflexões reforçam a importância de classificações alimentares que respeitem e possibilitem escolhas individuais, considerando valores, práticas e demais aspectos socioculturais que influenciam os padrões alimentares ${ }^{28}$.

Ainda em relação a distintas possibilidades de classificação dos alimentos, considera-se importante mencionar a atual discussão lançada por Monteiro et al. ${ }^{29}$, que propõe que os alimentos sejam classificados com base na extensão e no propósito do processamento industrial empregado na sua produção. O objetivo dos autores é alertar a comunidade científica e a população sobre os riscos relacionados ao consumo excessivo de alimentos industralizados prontos ou semiprontos para o consumo - ultraprocessados -, que, em sua maioria, apresentam excessiva quantidade de calorias. Nessa categoria se incluem muitos dos itens comercializados em bares, cantinas e ambulantes ao redor das escolas, os quais requerem mínima ou nenhuma preparação e têm longos prazos de validade. Vale destacar que a proposta de Monteiro et al. ${ }^{29}$ não objetiva contrapor as classificações baseadas no perfil nutricional dos alimentos, mas sim atentar para a necessidade de se resgatarem hábitos alimentares cultural- 
mente tradicionais. Para isso, tornam-se fundamentais medidas públicas que intervenham na qualidade dos alimentos disponíveis para consumo.

Atualmente, no Distrito Federal, existem legislações locais que estabelecem diretrizes para a segurança alimentar e nutricional e que, dentre outros aspectos, determinam os alimentos permitidos e os proibidos dentro do ambiente escolar. Apesar de essas normativas representarem uma tentativa de apoio na condução de estratégias voltadas para a promoção da alimentação saudável nas escolas, verifica-se ainda que essas ações são incipientes, principalmente com relação à cantina escolar. No Distrito Federal, observa-se um registro formal de ações específicas para a cantina escolar apenas dentre as ações desenvolvidas pelo Projeto A Escola Promovendo Hábitos Alimentares Saudáveis, uma das linhas de pesquisa do Observatório de Políticas de Segurança Alimentar e Nutrição, existente desde $2001^{12,13,30}$.

Escola e cantina escolar devem ser polos irradiadores de conhecimentos, atitudes e práticas que promovam e que estimulem a prática alimentar saudável, influenciando na formação de hábitos alimentares saudáveis dos alunos e da comunidade escolar e contribuindo para a garantia do direito humano à alimentação adequada e, consequentemente, para a melhoria da saúde da população escolar.

\section{ON CLUSÃ O}

Diante do exposto, reforça-se que a proposta metodológica desenvolvida para a implantação da cantina escolar saudável mostrou potencial para estimular e apoiar o desenvolvimento de uma alimentação saudável dentro do espaço da cantina escolar. Isso foi evidenciado por uma maior aplicação dos Dez Passos da Cantina Escolar Saudável e pelo aumento da qualidade dos lanches ofertados, do $1^{\circ}$ para o $2^{\circ}$ momento de avaliação. Apesar disso, o $3^{\circ}$ momento de avaliação identificou a não sustentabilidade de algumas ações referentes à manutenção dos Dez
Passos para a Cantina Saudável e da oferta de lanches mais saudáveis.

Portanto, para a efetiva implantação e manutenção da cantina escolar saudável, é fundamental o acompanhamento sistemático desse processo, apoiado no envolvimento da comunidade escolar e em uma legislação específica para as cantinas escolares. Essas três dimensões, quando contempladas, representarão um avanço concreto para o alcance do direito humano à alimentação adequada no ambiente escolar.

\section{A GRADECIMENTOS}

Ao corpo discente de graduação e pós-graduação que participou da pesquisa, ao departamento de Nutrição e aos proprietários de cantinas escolares. Ao Ministério da Saúde pelo financiamento.

\section{COLABORADORES}

NFA AMORIM, BAS SCHMITZ e MLCF RODRIGUES contribuíram com a concepção, desenvolvimento da pesquisa, análise dos dados, redação do artigo e revisão da versão final. EIG RECINE contribuiu com a orientação da análise dos dados, redação do artigo e revisão de sua versão final. CG GABRIEL contribuiu com a redação do artigo e revisão da versão final.

\section{REFERÊ NCIAS}

1. Lobstein $T$, Baur L, Uauy R. Obesity in children and young people: a crisis in public health. Obes Rev. 2004; 5(Suppl 1):s4-s85. doi: 10.1111/j.1467-78 9X.2004.00133.x.

2. Brasil. Ministério da Saúde. Portaria Interministerial $n^{\circ} 1.010$, de 8 de maio de 2006. Institui as diretrizes para a promoção da alimentação saudável nas escolas de educação infantil, fundamental e nível médio das redes públicas e privadas, em âmbito nacional. Diário Oficial da União. 9 maio 2006.

3. Schmitz BAS, Corso ACT, Caldeira GV, Gimeno SGA, Gabriel CG, Vasconcelos FAG. Overweight and obesity related factors in schoolchildren in Santa Catarina State, Brazil. Arch Latinoam Nutr. 2010; 60(4):332-9.

4. Ippolito-Shepherd J, Cerqueira MT, Ortega DP. Iniciativa Regional Escuelas Promotoras de la Salud 
en las Américas. Glob Health Promot. 2005; 12(3-4):220-9. doi: 10.1177/1025382305012003 0139.

5. Gabriel CG, Santos MV, Vasconcelos FAG, Milanez GHG, Hulse SB. Cantinas escolares de Florianópolis: existência e produtos comercializados após a instituição da Lei de Regulamentação. Rev Nutr. 2010; 23(2):191-9. doi: 10.1590/S1415-5273 2010000200002.

6. Bell AC, Swinburn BA. What are the key food groups to target for preventing obesity and improving nutrition in schools? Eur J Clin Nutr. 2004; 58(2):258-63. doi:10.1038/sj.ejcn.1601775.

7. Kramer-Atwood JL, Dwyer J, Hoelscher DM, Nicklas TA, Johnson RK, Schulz G. Fostering healthy food consumption in schools: focusing on the challenges of competitive foods. J Am Diet Assoc. 2002; 102(9):1228-33. doi:10.1016/S0002-8223(02)90 272-5.

8. Story M, Hayes M, Kalina B. Availability of foods in high schools: is there cause for concern? J Am Diet Assoc. 1996; 96(2):123-6. doi: 10.1016/S0002-82 23(96)00039-9.

9. Gabriel CG, Vasconcelos FA, Andrade DF, Schmitz BAS. First Law regulating school canteens in Brazil: evaluation after seven years of implementation. Arch Latinoam Nutr. 2009; 59(2):128-38.

10. Brasil. Manual das cantinas escolares saudáveis: promovendo a alimentação saudável. Brasília: Ministério da Saúde; 2010 [acesso 2011 jun 13]. Disponível em: <http://189.28.128.100/nutricao/docs/ geral/manual_cantinas.pdf>.

11. Brasil. Experiências estaduais e municipais de regulamentação da comercialização de alimentos em escolas no Brasil: identificação e sistematização do processo de construção e dispositivos legais adotados. Brasília: Ministério da Saúde; 2007 [acesso 2011 jun 22]. Disponível em: <http://189. 28.128.100/nutricao/docs/geral/regula_comerc_ alim_escolas_exper_estaduais_municipais.pdf $>$.

12. Rodrigues MLF, Silva JRM, Bernardon R, Amorim $\mathrm{N}$, Recine E, Monteiro R, et al. A escola promovendo hábitos alimentares saudáveis: uma estratégia educacional para a promoção da alimentação saudável na rede de ensino infantil e fundamental. In: Tecendo redes: conexão entre saberes para a educação. Rio de Janeiro: e-papers; 2007. p.61-81.

13. Schmitz BAS, Recine E, Cardoso GT, Silva JRM, Amorim NFA, Bernardon R, Rodrigues MLCF. A escola promovendo hábitos alimentares saudáveis: uma proposta metodológica de capacitação para educadores e donos de cantina escolar. Cad Saúde Pública. 2008; 24(Supl 2):s312-s22. doi:10.1590/S0 102-311X2008001400016.
14. Center for Disease Control and Prevention. U.S. Department of Health and Human Services. Guidelines for School Health Programs to Promote Lifelong Healthy Eating. MMWR Recomm Rep. 1996; 45 (RR-9): 1-33.

15. Food and Drug Administration. Code of Federal Regulations, Title 21 - Food and drugs (v. 2), Chapter I-Food and Drug Administration, Department of Health and Human Services, Part 101-Food Labeling-Table of Contents, Subpart AGeneral Provisions, Sec. 101.14 Health claims: general requirements and Subpart E-Specific requirements for Health Claims. U.S. Government Printing Office, Revised as of April 1. 2002.

16. Food And Drug Administration. Code of Federal Regulations, Title 21 - Food and drugs (v. 2), Chapter I- Food and Drug Administration, Department of Health and Human Services, Part 101-Food Labeling-Table of Contents, Subpart AGeneral Provisions, Sec. 101.12 Reference amounts customarily consumed per eating occasion. Silver Spring (MD): FDA; 2002

17. Akutsu RC, Botelho RA, Camargo EB, Sávio KEO, Araújo WC. The technical cards as quality instrument for good manufacturing process. Rev Nutr. 2005; 18(2):277-9. doi: 10.1590/\$1415-52 732005000200011.

18. Brasil. Dez passos para a promoção da alimentação saudável nas escolas. Brasília: Ministério da Saúde; 2004 [acesso 2011 jun 22]. Disponível em: <http:// 189.28.128.100/nutricao/docs/geral/dezPassos PasEscolas.pdf>.

19. French SA. Public health strategies for dietary change: schools and workplaces. J Nutr. 2005; 135(4):910-2.

20. Müller MJ, Danielzik S, Pust S. School and familybased interventions to prevent overweight in children. Proc Nutr Soc. 2005; 64(2):249-54.

21. Sharman M. International school-based interventions for preventing obesity in children. Obes Rev. 2006; 8(2):155-67. doi: 10.1111/j.1467-789X.2006.002 68.x.

22. Mâsse LC, Frosh MM, Chriqui JF, Yaroch AL, AgursCollins T, Blanck HM, et al. Development of a School Nutrition-Environment State Policy Classification System (SNESPCS). Am J Prev Med. 2007; 33(Suppl 1):s277-s291. doi:10.1016/j.amepre.2007.07.017.

23. Guia alimentar para a população brasileira. Brasília: Ministério da Saúde; 2006 [acesso 2011 jun 22]. Disponível em: <http://dtr2001.saude.gov.br/ editora/produtos/livros/pdf/05_1109_M.pdf>.

24. Ledikwe JH, Ello-Martin J, Rolls BJ. Portion sizes and the obesity epidemic. J Nutr. 2005; 135(4): 905-9. 
25. Mendonça CP, Anjos LA. Aspectos das práticas alimentares e da atividade física como determinantes do crescimento do sobrepeso/obesidade no Brasil. Cad Saúde Pública. 2004; 20 (Supl 3): 698-709. doi: 10.1590/S0102-311X200400030 0006.

26. Labouze E, Goffi C, Moulay L, Azaïs-Braesco V. A multipurpose tool to evaluate the nutritional quality of individual foods: Nutrimap. Public Health Nutr. 2007; 10(7):690-700. doi: 10.1017/S1368980007 382505.

27. Vieira-da-Silva LM, Almeida Filho N. Eqüidade em saúde: uma análise crítica de conceitos. Cad Saúde Pública. 2009; 25(Supl 2):s217-s26. doi: 10.1590/S 0102-311X2009001400004.

28. Garcia RWD. Práticas e comportamento alimentar no meio urbano: um estudo no centro da cidade de São Paulo. Cad Saúde Pública. 1997; 13(3): 455-67. doi: 10.1590/S0102-311X1997000300 021.

29. Monteiro CA, Levy RB, Claro RM, Castro IRR, Cannon G. A new classification of foods based on the extent and purpose of their processing. Cad Saúde Pública. 2010; 26(11):2039-49. doi: 10.15 90/S0102-311X2010001100005.

30. Bernardon R, Silva JRM, Cardoso GT, Monteiro RA, Amorim NFA, Schmitz BAS, et al. Construção de metodologia de capacitação em alimentação e nutrição para educadores. Rev Nutr. 2009; 22(3): 389-98. doi: 10.1590/S1415-5273200900030 0009.

Recebido em: 28/9/2011

Versão final em: 16/3/2012

Aprovado em: 23/3/2012 
